\title{
Eliminación de Enterococcus faecalis por medio del uso de hipoclorito de sodio, clorhexidina y MTAD en conductos radiculares
}

\section{Elimination of Enterococcus faecalis using sodium hypochlorite, chorhexidine and MTAD in root canals}

Pupo Marrugo S***, Díaz Caballero A**, Castellanos Berrio P*, Simancas Escorcia V*

\section{RESUMEN}

Objetivos: Comparar la efectividad de diferentes soluciones irrigadoras en la eliminación de cepas de Enterococcus faecalis en pacientes con patología periapical crónica, mediante pruebas microbiológicas.

Métodos: Se evaluaron 21 dientes con diagnóstico de periodontitis apicales crónicas no supurativas de pacientes que asistieron a consulta en las clínicas odontológicas de la Universidad de Cartagena, previa firma y aprobación del consentimiento informado para participar en el estudio. Los sujetos de estudio se asignaron aleatoriamente en tres grupos usando las siguientes sustancias irrigantes: hipoclorito de sodio al 5\%, clorhexidina al 2\% e hipoclorito de sodio 2,5\% con irrigación final de MTAD. Se identificaron microorganismos por medio de la prueba Reacción en Cadena de la Polimerasa (PCR) y se cuantificaron las unidades formadoras de colonias de Enterococcus faecalis antes y después de ser utilizadas las sustancias irrigadoras. Análisis estadístico: Se realizó el test de Kruskal wallis.

Resultados: La investigación demostró que todas las sustancias fueron efectivas en la eliminación de $E$. faecalis en pacientes con periodontitis apicales crónicas no supurativas. El hipoclorito de sodio al $5 \%$ ( $\mathrm{p}=$ $0,018)$, hipoclorito de sodio y MTAD $(p=0,021)$ y clorhexidina al $2 \%(p=0,028)$ fueron igual de efectivas. Conclusiones: El hipoclorito de sodio al $5 \%$, clorhexidina al $2 \%$, hipoclorito de sodio y MTAD pueden ser utilizadas en pacientes con periodontitis apical crónica no supurativa por ser efectivas en la eliminación de $E$. faecalis.

Palabras clave: Periodontitis periapical, hipoclorito de sodio, clorhexidina, Enterococcus faecalis (Decs Bireme).

\section{SUMMARY}

Objectives: To compare the effectiveness of different irrigating solutions in eliminating of Enterococcus faecalis in patients with chronic periapical pathology by applying microbiological tests.

Methods: Twenty one teeth with chronic apical periodontitis of non suppurative diagnosis of patients that attended the dental clinics of the University of Cartagena were evaluated after the patients signed the consent form to participate in the study. The study subjects were randomized into three groups using the following irrigating substances: Sodium hypochlorite at $5 \%$, chlorhexidine $2 \%$ and sodium hypochlorite and MTAD.

* $\quad$ Odontólogo de la Facultad de Odontología. Universidad de Cartagena (Colombia).

** Odontólogo en la Universidad de Cartagena. Especialista en Periodoncia en la Universidad Javeriana. Magíster en Educación, Universidad del Norte. PhD (Cand) en Ciencias Biomédicas, Universidad de Cartagena. Profesor titular, Facultad de Odontología. Universidad de Cartagena (Colombia).

*** Odontóloga y Endodoncista de la Universidad de Cartagena. Docente de pregrado y postgrado de la Universidad de Cartagena (Colombia). 
Microorganisms were identified through the Chain Reaction (PCR) and quantified the colony forming units before and after Enteroccocus faecalis be used irrigating substances for cleansing. Statistical analysis: We performed the Kruskal Wallis test.

Results: The research showed that all substances were effective in eliminating $E$. faecalis in patients with chronic apical periodontitis non suppurative. Sodium hypochlorite $5 \%(p=0,018)$, sodium hypochlorite and MTAD ( $p=0,021)$ and chlorhexidine $2 \%(p=0,028)$ were equally effective.

Conclusions: Sodium hypochlorite $5 \%$ chlorhexidine $2 \%$ and sodium hypochlorite and MTAD can be used in patients with chronic apical periodontitis non suppurative after being proven effective in eliminating $E$. faecalis.

Key words: Periapical periodontitis, sodium hypochlorite, chlorhexidine, Enterococcus faecalis (Decs Bireme).

Fecha de recepción: 22 de febrero de 2014.

Aceptado para publicación: 5 de marzo de 2014.

Pupo Marrugo S, Díaz Caballero A, Castellanos Berrio P, Simancas Escorcia V. Eliminación de Enterococcus faecalis por medio del uso de hipoclorito de sodio, clorhexidina y MTAD en conductos radiculares. Av. Odontoestomatol 2014; 30 (5): 263-270.

\section{INTRODUCCIÓN}

El objetivo fundamental del tratamiento endodóntico, es la eliminación de los microorganismos (1). En casos de Periodontitis Apical Crónica No Supurativa (PACNS) la terapia del canal radicular a través de la preparación quimiomecánica busca eliminar el material orgánico, inorgánico y los microorganismos presentes en el conducto. Se espera que luego de la realización del tratamiento de conductos, se desencadene un proceso de cicatrización periapical, que conducirá a la resolución de la lesión. Diversas investigaciones demuestran que el Enterococcus faecalis es uno de los responsables de los fracasos endodónticos en los casos de PACNS, además de ser resistente a la acción de numerosas sustancias irrigantes, su permanencia en el conducto radicular está asociado, por un lado, a la capacidad de sobrevivir en ambientes hostiles y, por otro, a la formación de un biofilm con otros microorganismos. Actualmente, se usan diversos tipos de sustancias irrigantes para la eliminación del Enterococcus faecalis pero aún no se encuentra la ideal (2).

El hipoclorito de sodio de 0,5-6\% (NaOCl), se considera la solución irrigadora más utilizada en la práctica clínica endodóntica, por ser la que más se acerca a las condiciones ideales por su efectividad para eliminar tejido vital y no vital, además de poseer un amplio efecto antibacteriano, matando rápidamente bacterias, esporas, hongos y virus. Sus concentraciones clínicas varían entre el $0,5 \%$ al $6 \%$, con la dilución del $\mathrm{NaOCl}$ disminuye significativamente la propiedad antibacteriana al igual que disminuye su toxicidad (3).

La clorhexidina, es un compuesto catiónico antibacteriano como irrigante endodóntico es utilizado al $0,12 \%$ o $2 \%$, las propiedades antibacterianas son similares al $\mathrm{NaOCl}$ e incluso tiene mejor efecto residual a las 24 horas, pero no tiene la capacidad de disolver tejido pulpar (4).

Actualmente, se dispone de una nueva sustancia irrigadora, el MTAD, solución ácida con $\mathrm{pH}$ de 2,15 , es una mezcla de un isómero de tetraciclina (doxiciclina), un ácido (ácido cítrico) y un detergente (Tween). Su principal acción es la remoción efectiva del barrillo dentinario, posterior al empleo de $\mathrm{NaOCl}$. Las tetraciclinas son de naturaleza bacteriostática, presentan un $\mathrm{pH}$ bajo, poseen capacidad de quelar calcio y tienen actividad anticolagenasa, lo que confiere al MTAD sus efectos antibacterianos. Singla et al. demostró que el MTAD es capaz de eliminar la capa de barrillo dentinal y ser eficaz contra el Enterococcus faecalis. Sin embargo, no pudo simular un ambiente clínico y sugiere se realicen más estudios para determinar el efecto del MTAD 
en la infección bacteriana a nivel de los túbulos dentinarios (5).

Luego, es importante estudiar la efectividad de las soluciones irrigadoras tradicionales como; el hipoclorito de sodio y alternativas como; clorhexidina y el MTAD en la eliminación de Enterococcus faecalis en conductos radiculares de pacientes con periodontitis apicales crónicas con el propósito de analizar y establecer cuál es la terapéutica adecuada.

\section{MATERIALES Y MÉTODOS}

Estudio clínico experimental. Se evaluaron 21 dientes en 19 pacientes con periodontitis apicales crónicas no supurativas, seleccionados a partir de los siguientes criterios: se incluyeron sujetos con diagnóstico clínico y radiográfico de periodontitis apical crónica no supurativa en dientes unirradiculares con conducto permeable no tratados endodónticamente, que asistieran a consulta en las clínicas odontológicas de la Universidad de Cartagena. Fueron excluidos individuos con enfermedades sistémicas no controladas y que estuvieran siendo tratados con antibióticos y corticosteroides.

En esta investigación prevaleció el respeto, dignidad, bienestar, protección de los derechos del paciente, y consideraciones bioéticas contempladas en la Declaración de Helsinki. El paciente, previa atención, firmó y aprobó a los investigadores un consentimiento informado donde se le explicó que se realizaba una investigación científica en seres humanos de riesgo mínimo a través de procedimientos comunes y no invasivos, quedando sujeto a todo lo dispuesto en la resolución 008430 de 1993 del Ministerio de Salud de Colombia.

Las mediciones principales de la variable respuesta en el presente estudio fueron: identificación de microorganismos a través de PCR y la cuantificación de unidades formadoras de colonias de Enterococcus faecalis.

Los 21 conductos radiculares fueron distribuidos por asignación aleatoria simple, a tres grupos de estudio usando las siguientes sustancias irrigantes: hipoclorito de sodio (grupo $1=5$ sujetos), clorhexidi- na (grupo $2=7$ sujetos), hipoclorito de sodio y MTAD (grupo $3=7$ sujetos). En los sujetos tratados con hipoclorito de sodio al $5 \%$ y clorhexidina al $2 \%$ la Irrigación fue de $2 \mathrm{ml}$ entre cada lima, se preparó apicalmente con $5 \mathrm{ml}$ durante 60 segundos para cada lima a $1 \mathrm{~mm}$ de la longitud real de trabajo (LRT). Para los tratados con Hipoclorito de sodio y MTAD combinado, el procedimiento inicial fue igual que el primer grupo, pero en la preparación apical la irrigación final fue de $5 \mathrm{ml}$ de MTAD $1 \mathrm{~mm}$ de LRT durante 60 segundos.

\section{Procedimiento para la toma de muestras intracanales}

Se realizó profilaxis con solución salina, instrumental rotatorio de baja velocidad, copa de caucho y cepillo profiláctico a nivel coronal en el cuadrante del órgano dentario a tratar y a continuación aislamiento con tela de caucho, se seleccionó la grapa y se colocó la tela, el arco de Young y la grapa al mismo tiempo. Se realizó la apertura del conducto, con estrictas medidas de bioseguridad, con fresa de diamante redonda $\mathrm{n}^{\circ} 4$, se introdujo en los conductos radiculares una lima estéril tipo Hedstrom 08 hasta una longitud de $1 \mathrm{~mm}$ del ápice radiográfico, se realizó un limado sobre las paredes del conducto radicular, hasta obtener una cantidad considerable de barrillo dentinal. Luego se tomó una muestra con puntas de papel esterilizado $n^{\circ} 15$, que permaneció en el conducto radicular por 20 segundos. La entrada de los conductos fue sellada después de la instrumentación e irrigación, en forma hermética con resina compuesta de foto polimerizado, evitando de esta manera la sobreinfección del mismo.

Estas muestras fueron almacenadas en tubos eppendorf con 500 microlitros de solución buffer TE (Tris - EDTA, pH 8,0) y transportadas al laboratorio de microbiología de la Facultad de Medicina de la Universidad de Cartagena en donde fueron inmediatamente cultivadas. A los siete días siguientes el sujeto de estudio fue citado para la segunda toma de muestra en los canales radiculares, la cual se llevó a cabo de la forma anteriormente descrita. Se realizó la reinstrumentación y preparación apical y finalmente se obturaron los conductos radiculares con la técnica de condensación lateral (6). 


\section{Procesamiento de las muestras}

En el laboratorio de microbiología cada muestra se agitó en un mezclador Vortex para homogeneizar bien la muestra, máxima velocidad por 30 segundos. Luego se tomaron $20 \mu \mathrm{L}$ de la solución bacteriana y se inocularon en una placa de agar selectivo para Enterococcus faecalis (ChromID CPS3, Biomeriux). Posteriormente se incubaron en un ambiente de microaerofilia a $37^{\circ} \mathrm{C}$ por 48 horas. Se observó crecimiento de colonias de color verde y bordes definidos para $E$. faecalis. Se realizó recuento de unidades formadoras de colonias (UFC), y pruebas de reacción en cadena de la polimerasa (PCR) para la identificación final de los microorganismos aislados. Los cultivos que eran negativos en las primeras 48 horas eran incubados hasta 96 horas, para determinar que la muestra era negativa. Los aislamientos positivos fueron almacenados a $-80^{\circ} \mathrm{C}$ para su posterior identificación por PCR.

\section{Identificación molecular por PCR}

La extracción del ADN bacteriano se realizó a partir de las colonias obtenidas de un cultivo de 24 horas. Las colonias fueron suspendidas en $500 \mu \mathrm{L}$ de buffer TE (1 mM EDTA. 10 mMTris-HCl-pH 8,0). Luego, la suspensión bacteriana era calentada a $99^{\circ} \mathrm{C}$ por 30 minutos. Se centrifugaba a $13.000 \times g$ por 15 minutos y, finalmente, $5 \mu \mathrm{L}$ del sobrenadante eran utilizados para las reacciones de PCR. La PCR múltiple de todos los aislados se llevó a cabo utilizando tres juegos de oligonucleótidos cebadores descritos en la literatura, obtenidos de: EntG-F: 5 “ TACTGACAAACCATTCATGATG'3 y EntG-R: 5'AACTTCGTCACCAAC GCGAAC'3 que amplifica un fragmento de 112 pares de bases (pb) del gen tuf, específico para identificar el género Enterococcus; Efaeca-F : 5‘ATCAAGTACAGTTAG TCT'3 Efaeca-R: 5'ACGATTCAAAGCTAACTG' 3 que amplifica un fragmento de $941 \mathrm{pb}$ del gen ddl específico de Enterococcus faecalis. El ADN fue amplificado en un volumen de reacción de $25 \mu \mathrm{L}$ que contenía $12,5 \mu \mathrm{L}$ de la mezcla de PCR (PCR master Mix; Promega), 0,2 $\mu \mathrm{M}$ de cada cebador y $5 \mu \mathrm{L}$ de ADN molde. La reacción se llevó a cabo en un termociclador Perkin-Elmer bajo las siguientes condiciones: un ciclo inicial de desnaturalización a $94^{\circ} \mathrm{C}$ por 5 minutos (min), se- guido por 35 ciclos de $94^{\circ} \mathrm{C}$ por $1 \mathrm{~min}, 50^{\circ} \mathrm{C}$ por 1 min, y $72^{\circ} \mathrm{C}$ por $2 \mathrm{~min}$, con un ciclo de extensión final a $72^{\circ} \mathrm{C}$ por $5 \mathrm{~min}$. Como controles se utilizó la cepa de Enterococcus faecalis ATCC 33591. Todos los productos amplificados fueron visualizados en Gel de agarosa al $2,0 \%$ con bromuro de etidio $0,5 \mu \mathrm{g} / \mathrm{ml}$, mediante un transiluminador de luz UV.

\section{Análisis estadístico}

Los resultados obtenidos fueron expresados estadísticamente de la siguiente forma: de cada muestra se tomó un dato del recuento inicial y otro final de UFC de Enterococcus faecalis. La evaluación de las sustancias utilizadas fue cuantitativa, se obtuvieron los datos con las tres sustancias en la eliminación del Enterococcus faecalis y se analizaron con la prueba de Kruskal Wallis. Para comparar los resultados obtenidos entre las tres sustancias se realizó el test de Wilcoxon.

\section{RESULTADOS}

Las muestras de estudio fueron tomadas de 21 conductos radiculares, divididas en tres grupos de 7 conductos que corresponden a 33,3\% de la muestra total. El recuento inicial de las unidades formadoras de colonias (UFC) para las muestras tratadas con hipoclorito de sodio fue de 82,5 y las finales de 78 UFC. Para la clorhexidina las UFC fueron 82,5 en el recuento inicial y en el recuento final fue de 96,5. Los valores de UFC iniciales para el grupo de hipoclorito de sodio y MTAD fue de 66 y de 56,5 para el recuento final. Estas diferencias entre los tres grupos no fueron estadísticamente significativas. Esta investigación demostró que el hipoclorito de sodio $(\mathrm{p}=$ $0,018)$, clorhexidina $(p=0,028)$ e hipoclorito de sodio y MTAD $(p=0,021)$ fueron efectivas en la eliminación de $E$. faecalis en pacientes con periodontitis apicales crónicas no supurativas.

Con el fin de determinar si los microorganismos aislados correspondían a $E$. faecalis se llevaron a cabo pruebas moleculares a través de la reacción en cadena de la polimerasa (PCR) (7), usando tres juegos de oligonucleótidos cebadores, obtenidos de: EntGF: 5' TACTGACAAACCATTCATGATG'3 y EntG-R: 
5'AACTTCGTCACCAAC GCGAAC'3 que amplifica un fragmento de 112 pares de bases (pb) del gen tuf, específico para identificar el género Enterococcus; Efaeca-F: 5‘ATCAAGTACAGTTAG TCT'3 Efaeca-R: 5'ACGATTCAAAGCTAACTG'3 que amplifica un fragmento de 941 pb del gen ddl especifico de Enterococcus faecalis. El ADN fue amplificado en un volumen de reacción de $25 \mu \mathrm{L}$ que contenía $12,5 \mu \mathrm{L}$ de la mezcla de PCR (PCR master Mix; Promega), 0,2 $\mu \mathrm{M}$ de cada cebador y $5 \mu \mathrm{L}$ de ADN molde. La reacción se llevó a cabo en un termociclador PerkinElmer. Se determinó que el $100 \%$ de las muestras analizadas por PCR correspondían al Enterococcus faecalis. Lo que indica que la PCR logro identificar específicamente el agente causal de la lesión en aquellos individuos con cultivos positivos.

\section{DISCUSIÓN}

El papel de la instrumentación endodóntica es la reducción de las poblaciones de bacterias a nivel intracanal, que, sin embargo necesita ser complementada con el uso de sustancias irrigantes antibacterianas basado en un enfoque quimiomecánico (810). El presente estudio fue llevado a cabo con el fin de comparar la eficacia antibacteriana de tres sustancias irrigantes; el hipoclorito de sodio, clorhexidina y MTAD durante la preparación quimiomecánica en la eliminación de las poblaciones intracanales de Enterococcus faecalis, microorganismo resistente a las condiciones extremas y que, con frecuencia, se encuentra en los casos de fracaso endodóntico $(2,11)$. La eficacia de las sustancias irrigadoras se determinó a través de la medición en la reducción de UFC a partir de dos muestras tomadas por conducto radicular, una inicial y otra siguiente a la irrigación, en pacientes diagnosticados con periodontitis apicales crónicas.

$\mathrm{El} E$. faecalis es una de las principales especies bacterianas aisladas en las muestras de infecciones endodónticas debido a su capacidad de invadir los túbulos dentinarios y adherirse a las paredes del conducto radicular mediada por componentes celulares específicos (12) y As (colágeno-proteína de unión a los Enterococcus) (13). Además, algunos componentes de la dentina, como el colágeno tipo I, puede actuar como factor de selección para la adhe- sión bacteriana y la invasión (14). De esta forma, el $E$. faecalis puede persistir dentro de los túbulos dentinarios después del tratamiento endodóntico (15). Este estudio mostró un crecimiento de E. faecalis en la mayoría de las muestras, coincidiendo con un reciente estudio realizado por Williamson y col. (16), donde se informó que el sistema de conductos radiculares de los dientes con lesión perirradicular fueron significativamente más propensos a tener bacterias, comparados con los dientes sin lesión perirradicular, hallándose que las especies de Enterococcus se asociaron más con los dientes con lesiones perirradiculares y demostraron ser capaces de resistir los cambios de $\mathrm{pH}$ altamente básico, así como periodos prolongados con pocos o ningún nutrientes. Por su parte, Shabahang y col., en 2003 (17), mencionan que el $E$. faecalis es frecuentemente el patógeno dominante, a veces el único, en la periodontitis apical persistente, sugiriendo que está sola especie tiene el potencial para mantener una infección del conducto radicular y la lesión perirradicular. En la mayoría de los trabajos se encontró que el $E$. faecalis era el patógeno dominante $(2,18,19)$.

En cuanto a la reducción del E. faecalis, el hipoclorito de sodio al $5 \%$ en el presente estudio fue mayor al 50\%. Se comparó la proporción de eliminación de microorganismos con estudios realizados por Giardino y col. en el 2007 (20), donde demostraron que el hipoclorito de sodio al $5 \%$ es altamente eficaz en la erradicación de colonias de E. faecalis. Estos resultados son consistentes con trabajos publicados anteriormente utilizando diferentes metodologías $(16,17,21)$. El grupo de hipoclorito de sodio y MTAD en este estudio obtuvo una eliminación significativa de la mayoría de las UFC de E. faecalis, la eficacia de este grupo coincide con estudios hechos por Shabahang y col. en 2003 (17), donde demuestra un alto nivel de sensibilidad de E. faecalis al MTAD, incluso cuando esta solución se diluye 200 veces, este efecto es logrado gracias a la eliminación de la capa de barrillo dentinal y al efecto en la desinfección del canal radicular, eliminando bacterias incrustadas en dicha capa $(22,23)$.

El presente estudio mostró que el grupo del clorhexidina obtuvo una disminución cercana a la mitad de UFC de E. faecalis, resultando la menos eficaz. Este 
resultado difiere con estudios hechos por Ercan y col. (24) quienes hallaron una eficacia del $64 \%$ en los tratamientos realizados con clorhexidina, considerándola más eficaz que otras sustancias intracanales como el hidróxido de calcio en la eliminación de infección por $E$. faecalis dentro de los túbulos dentinarios, y la sugiere como un irrigante en la terapia endodóntica. Por su parte, Abdullah y col. (22), en el 2005, demostraron que la clorhexidina es un potente agente antimicrobiano, aunque no fue capaz de erradicar el $E$. faecalis. Este hallazgo es consistente con investigaciones previas $(16,17,25,26)$ y apunta a la necesidad de utilizar clorhexidina junto con otro agente químico que mejore su eficacia.

En este estudio se encontró efectividad en la irrigación con hipoclorito de sodio al $5 \%$, clorhexidina al $2 \%$ e hipoclorito de sodio y MTAD al eliminar las cepas de E. faecalis. A diferencia de Gomes y col. (26), en el año 2009 en el cual se aprecia que el $\mathrm{NaOCl} 2,5 \%$ y clorhexidina $2 \%$ gel no fueron efectivas en la eliminación de la endotoxina de los conductos radiculares infectados. Wang y col. (27) mostraron una reducción estadísticamente significativa del grupo de hipoclorito de sodio y MTAD e hipoclorito de sodio al 5,25\% en la eliminación del $E$. faecalis. La sustantividad de la doxiciclina es una propiedad importante en la eficacia del MTAD (28).

La identificación de microorganismos a través de la técnica de biología molecular PCR fue confirmatoria de la presencia de $E$. faecalis en los conductos radiculares de los dientes tratados en este estudio. Actualmente en microbiología la precisión de las metodologías diagnosticas son demasiado limitados para respaldar conclusiones definitivas debido:

1. Más del $50 \%$ de la microflora endodóntica está todavía sin cultivar $(29,30)$.

2. La no supervivencia de bacterias debido a su falta de crecimiento en medios de cultivo (31).

3. Las técnicas de toma de muestra son limitadas en cuanto a la obtención de las bacterias en el espacio del conducto radicular.

4. El riesgo de resultados falsos positivos.

Este estudio aporta una alternativa eficaz en la terapéutica de casos de periodontitis apicales crónicas; se propone el uso combinado de hipoclorito de sodio y MTAD. Sin embargo hay limitantes en el estu- dio y los resultados indican que se debe continuar en la búsqueda de la sustancia irrigadora ideal aún cuando se cuenta con sustancias altamente eficaces.

\section{CONCLUSIÓN}

El hipoclorito de sodio y MTAD resultan altamente eficaces en la eliminación de $E$. faecalis y puede ser usada en el tratamiento con periodontitis apical crónica no supurativa.

\section{AGRADECIMIENTOS}

Al personal del laboratorio de Microbiología de la Facultad de Medicina, a la Facultad de Química y Farmacia de la Universidad de Cartagena Colombia, por su colaboración en la realización de este trabajo.

\section{BIBLIOGRAFÍA}

1. Kennedy J, Hussey D. The antimicrobial effects of root canal irrigation and medication. Oral Surgery, Oral Medicine, Oral Pathology, Oral Radiology, and Endodontology 2007;103(4):5609.

2. Kayaoglu G, Ørstavik D. Virulence factors of Enterococcus faecalis: relationship to endodontic disease. Critical Reviews in Oral Biology $\&$ Medicine 2004;15(5):308-20.

3. Siqueira Jr JF, Rôças IN, Paiva SS, GuimarãesPinto T, Magalhães KM, Lima KC. Bacteriologic investigation of the effects of sodium hypochlorite and chlorhexidine during the endodontic treatment of teeth with apical periodontitis. Oral Surgery, Oral Medicine, Oral Pathology, Oral Radiology, and Endodontology 2007;104(1):12230.

4. Zamany A, Safavi K, Spångberg LS. The effect of chlorhexidine as an endodontic disinfectant. Oral Surgery, Oral Medicine, Oral Pathology, Oral Radiology, and Endodontology 2003;96(5):57881. 
5. Singla MG, Garg A, Gupta S. MTAD in endodontics: an update review. Oral Surgery, Oral Medicine, Oral Pathology, Oral Radiology, and Endodontology 2011;112 (3):e70-e6.

6. Gilhooly RM, Hayes SJ, Bryant ST, Dummer PM. Comparison of lateral condensation and thermomechanically compacted warm a-phase gutta-percha with a single cone for obturating curved root canals. Oral Surgery, Oral Medicine, Oral Pathology, Oral Radiology, and Endodontology 2001;91(1):89-94.

7. Fouad AF, Barry J, Caimano M, Clawson M, Zhu $Q$, Carver R, et al. PCR-based identification of bacteria associated with endodontic infections. Journal of clinical microbiology 2002;40(9):322331.

8. Byström A, Sundqvist G. Bacteriologic evaluation of the effect of 0.5 percent sodium hypochlorite in endodontic therapy. Oral Surgery, Oral Medicine, Oral Pathology 1983;55(3):307-12.

9. Siqueira Jr JF, Rôças IN, Santos SR, Lima KC, Magalhães FA, de Uzeda M. Efficacy of instrumentation techniques and irrigation regimens in reducing the bacterial population within root canals. Journal of Endodontics 2002; 28(3):181-4.

10. Siqueira Jr JF, Rôças IN, Favieri A, Lima KC. Chemomechanical reduction of the bacterial population in the root canal after instrumentation and irrigation with $1 \%, 2.5 \%$, and $5.25 \%$ sodium hypochlorite. Journal of Endodontics 2000;26(6): 331-4.

11. Siren E, Haapasalo M, Ranta K, Salmi P, Kerosuo E. Microbiological findings and clinical treatment procedures in endodontic cases selected for microbiological investigation. International Endodontic Journal 1997;30(2):91-5.

12. Waltimo T, Ørstavik D, Sirén E, Haapasalo M. In vitro yeast infection of human dentin. Journal of Endodontics 2000;26(4):207-9.

13. Stuart CH, Schwartz SA, Beeson TJ, Owatz CB. Enterococcus faecalis: Its Role in Root Canal
Treatment Failure and Current Concepts in Retreatment. Journal of endodontics 2006;32 (2):93-8.

14. Kowalski WJ, Kasper EL, Hatton JF, Murray BE, Nallapareddy SR, Gillespie MJ. Enterococcus faecalis. Adhesin, Ace, Mediates Attachment to Particulate Dentin. Journal of endodontics 2006; 32(7):634-7.

15. Kayaoglu G, Erten H, Bodrumlu E, Ørstavik D. The Resistance of Collagen-associated, Planktonic Cells of Enterococcus faecalis to Calcium Hydroxide. Journal of endodontics 2009;35(1): 46-9.

16. Williamson AE, Cardon JW, Drake DR. Antimicrobial Susceptibility of Monoculture Biofilms of a Clinical Isolate of Enterococcus faecalis. Journal of Endodontics 2009;35(1):95-7.

17. Shabahang S, Torabinejad M. Effect of MTAD on Enterococcus faecalis. -Contaminated Root Canals of Extracted Human Teeth. Journal of Endodontics 2003;29(9):576-9.

18. Turk BT, Sen BH, Ozturk T. In vitro antimicrobial activity of calcium hydroxide mixed with different vehicles against Enterococcus faecalis and Candida albicans. Oral Surgery, Oral Medicine, Oral Pathology, Oral Radiology, and Endodontology 2009;108(2):297-301.

19. Vianna ME, Gomes BP, Berber VB, Zaia AA, Ferraz CCR, de Souza-Filho FJ. In vitro evaluation of the antimicrobial activity of chlorhexidine and sodium hypochlorite. Oral Surgery, Oral Medicine, Oral Pathology, Oral Radiology, and Endodontology 2004;97(1):79-84.

20. Giardino L, Ambu E, Savoldi E, Rimondini R, Cassanelli C, Debbia EA. Comparative Evaluation of Antimicrobial Efficacy of Sodium Hypochlorite, MTAD, and Tetraclean Against Enterococcus faecalis. Biofilm. Journal of Endodontics 2007;33(7): 852-5.

21. Dunavant TR, Regan JD, Glickman GN, Solomon ES, Honeyman AL. Comparative Evaluation of 
Endodontic Irrigants against Enterococcus faecalis. Biofilms. Journal of Endodontics 2006;32 (6):527-31.

22. Abdullah M, Ng Y-L, Gulabivala K, Moles DR, Spratt DA. Susceptibilties of Two Enterococcus faecalis. Phenotypes to Root Canal Medications. Journal of endodontics 2005;31(1):30-6.

23. Torabinejad $M$, Handysides $R$, Khademi AA, Bakland LK. Clinical implications of the smear layer in endodontics: a review. Oral Surgery, Oral Medicine, Oral Pathology, Oral Radiology, and Endodontology 2002;94(6):658-66.

24. Ercan E, Dalli M, Türksel Dülgergil Ç, Yaman F. Effect of Intracanal Medication with Calcium Hydroxide and 1\% Chlorhexidine in Endodontic Retreatment Cases with Periapical Lesions: An In Vivo. Study. Journal of the Formosan Medical Association 2007;106(3):217-24.

25. Spratt D, Pratten J, Wilson M, Gulabivala K. An in vitro evaluation of the antimicrobial efficacy of irrigants on biofilms of root canal isolates. International Endodontic Journal 2001;34(4): 300-7.

26. Gomes BP, Martinho FC, Vianna ME. Comparison of $2.5 \%$ sodium hypochlorite and $2 \%$ chlorhexidine gel on oral bacterial lipopolysaccharide reduction from primarily infected root canals. Journal of Endodontics. 2009;35 (10):1350-3.

27. Wang J, Tauchi Y, Deguchi Y, Morimoto K, Tabata Y, Ikada Y. Positively charged gelatin microspheres as gastric mucoadhesive drug delivery system for eradication of H. pylori. Drug delivery 2000;7(4): 237-43.

28. Sjögren U, Figdor D, Persson S, Sundqvist G. Influence of infection at the time of root filling on the outcome of endodontic treatment of teeth with apical periodontitis. International Endodontic Journal 1997;30(5):297-306.

29. Shuping GB, Ørstavik D, Sigurdsson A, Trope M. Reduction of intracanal bacteria using nickeltitanium rotary instrumentation and various medications. Journal of Endodontics 2000;26 (12):751-5.

30. Siqueira J, Machado A, Silveira R, Lopes H, Uzeda Md. Evaluation of the effectiveness of sodium hypochlorite used with three irrigation methods in the elimination of Enterococcus faecalis from the root canal, in vitro. International Endodontic Journal 1997;30(4):279-82.

31. Weiger R, De Lucena J, Decker H, Löst C. Vitality status of microorganisms in infected human root dentine. International endodontic journal 2002;35(2):166-71.

\section{CORRESPONDENCIA}

Antonio Díaz Caballero

Facultad de Odontología. Grupo GITOUC Campus de Zaragocilla. Área de la Salud. Universidad de Cartagena.

Cartagena de Indias, D.T y C. Colombia.

Email: adiazc1@unicartagena.edu.co.

Web: www.unicartagena.edu.co. 